\title{
Rapid automatized naming as an index of genetic liability to autism
}

\author{
Molly Losh • Denise Esserman • Joseph Piven
}

Received: 10 October 2009 / Accepted: 1 April 2010 /Published online: 20 April 2010

(C) Springer Science+Business Media, LLC 2010

\begin{abstract}
This study investigated rapid automatized naming (RAN) ability in high functioning individuals with autism and parents of individuals with autism. Findings revealed parallel patterns of performance in parents and individuals with autism, where both groups had longer naming times than controls. Significant parent-child correlations were also detected, along with associations with language and personality features of the broad autism phenotype (retrospective reports of early language delay, socially reticent personality). Together, findings point towards RAN as a potential marker of genetic liability to autism.
\end{abstract}

Keywords Autism - Rapid automatized naming ·

Endophenotype $\cdot$ Broad autism phenotype

\section{Introduction}

A constellation of subtle personality, language, and behavioral features have been found to aggregate among

\author{
M. Losh $(\bowtie)$ \\ Department of Allied Health Sciences, \\ University of North Carolina, \\ Chapel Hill, NC 27599-7190, USA \\ e-mail: molly.losh@unc.edu \\ J. Piven \\ Carolina Institute for Developmental Disabilities, \\ University of North Carolina, \\ Chapel Hill, NC 27599, USA \\ D. Esserman \\ Department of Medicine, \\ University of North Carolina, \\ Chapel Hill, NC 27599, USA
}

unaffected relatives of autistic individuals, suggesting they may index genetic liability (for review see Losh et al. 2010). Studies of this broad autism phenotype (BAP) may constitute a promising avenue for identifying genetically meaningful features and neurobiological substrates involved in autism. However, as currently measured, the features of the BAP remain relatively removed from underlying cognitive mechanisms, hampering efforts to implement the BAP features in genetic and neurobiological studies.

In an attempt to further delineate the phenotype, recent research has begun to disaggregate the neuropsychological basis of the features of the BAP (e.g., Adolphs et al. 2008). In this vein, the present study examined rapid automatized naming ability (RAN) in individuals with autism and in parents. Parents of autistic individuals have been shown previously to exhibit slower times on RAN tasks (Piven and Palmer 1997), and elevated rates of related language processing difficulties (e.g., phonological encoding, readingrelated phenotypes) have also been reported (Bailey et al. 1995; Bolton et al. 1994; Folstein and Rutter 1977; Folstein et al. 1999; Hughes et al. 1999), although studies using different methodologies have produced conflicting results (e.g., Bishop et al. 2004; Pilowsky et al. 2003; Smalley and Asarnow 1990). This study sought to clarify whether RAN speed may serve as a genetically meaningful phenotype in autism, by studying rapid naming tasks previously shown to differentiate family members of individuals with autism from controls (i.e., color and object naming) in a large sample of parents of autistic individuals, as well as among a group of high-functioning individuals with autism, examining both group differences and parent-child correlations. Below, the significance of rapid naming ability is reviewed briefly followed by rationale for examining this ability in autism. 


\section{The significance of rapid naming ability}

The rapid automatized naming (RAN) task first appeared in The Mental Examiner's Handbook as a bedside measure of recovery from brain injury in the mid-1900s. Patients were shown a series of randomly repeating squares printed in primary colors and instructed to name the colors as quickly as possible, with speed and accuracy measured as indices of recovery. Since this time, RAN has emerged as a sensitive index of reading ability (Wolf et al. 2000), and is known to tap such fundamental language skills as phonological processing and memory, articulation, and linguistic processing speed (Denckla 1972; Denckla and Cutting 1999; Denckla and Rudel 1974, 1976; Holland et al. 2004).

Early studies of RAN in patients with focal brain lesions and more recent imaging studies have helped to clarify those brain regions involved in this skill, implicating the inferior frontal cortex, temporo-parietal areas, and the corpus callosum (Dougherty et al. 2007; Misra et al. 2004 ; Phinney et al. 2006). Complementary studies of RAN in children have also linked this ability to specific stages of brain development (Denckla 1972), with recent research showing that response speed measured by RAN is linked to myelin deposition over the course of development, and resulting advances in language related skills (Dougherty et al. 2007). Deficits in rapid naming have been associated with decreased connectivity (Deutsch et al. 2005; Mabbott et al. 2006). Given evidence of cortical underconnectivity in autism (Just et al. 2004, 2007; Kana et al. 2009), such findings lend support for the study of RAN ability as a possible neuroarchitectural marker relevant to autism. Finally, RAN ability is highly heritable (Byrne et al. 2002; Compton et al. 2001; Petrill et al. 2006) making this skill a good target for genetic studies. Indeed, genetic linkage and candidate gene studies of dyslexia (a disorder in which RAN is severely disrupted) have reported significant linkage and association to RAN ability for several genomic regions (de Kovel et al. 2008; Grigorenko et al. 1997; 2001; Nopola-Hemmi et al. 2002).

This study focused on RAN ability among relatives of individuals with autism, and high functioning individuals with autism in an attempt to replicate prior findings of RAN deficits among relatives (Piven and Palmer 1997), and highlight language-related phenotypes and associated underlying neural structures which may be influenced by autism susceptibility genes. Specifically, we aimed to tie our findings to the well-established neurocognitive literature on RAN to gain insights into brain regions which may be implicated in the language-related characteristics of autism. This study examined rapid naming in a group of over 300 parents of autistic individuals and 36 high-functioning individuals with autism, as well as age-and IQ-matched controls. Based on prior findings that parents of individuals with autism differed from controls on the color and object domains of RAN (Piven and Palmer 1997), and suggestion that the color and object domains of RAN are believed to be less influenced by experience/exposure to print than letter and number naming and therefore most appropriate for studies of genetic influence, only the color and object tasks were administered. Recent studies of RAN among individuals with autism and their parents using aggregate RAN scores on the Comprehensive Test of Phonological Processing indicated no significant differences with controls (Lindgren et al. 2009). We therefore examined the specific subtests of color and object naming shown to differentiate parents from controls in prior studies (Piven and Palmer 1997; Folstein et al. 1999).

Analyses examined group differences as well as parentchild correlations that could index genetic effects. Finally, exploratory analyses of associations between RAN and features of the BAP (social behavior, rigid/perfectionistic personality, and early language-related delays) were conducted. Prior work suggests that reading-related phenotypes among relatives (nonsense word reading) may be associated with retrospectively reported language-related delays, but not with other features of the BAP (Folstein et al. 1999). Our prior work has also shown that performance on tasks indexing neuropsychological function cosegregate specifically with features of the BAP (Losh et al. 2009), suggesting that it may be important to examine features of the BAP in relation to RAN ability as well. We therefore examined history of language delay, as well as other key features of the BAP, in relation to RAN to explore how this ability may inform knowledge of the underpinnings of the various BAP features.

\section{Methodology}

\section{Participants}

All three samples of autism families had one or more children meeting criteria for autistic disorder based on the following criteria: DSM-IV (APA 1994), Autism Diagnostic Interview-Revised (Lord et al. 1994), and Autism Diagnostic Observation Schedule (Lord et al. 2000). All children were screened for associated medical conditions (e.g., tuberous sclerosis or fragile $\mathrm{X}$ syndrome) and evidence of gross central nervous system injury and/or severe perinatal events. Sample characteristics are summarized in Table 1, and presented in detail below.

Parents The parent sample was comprised of three separately ascertained groups of families. The first consisted of 48 parents of multiple children with autism (multiplex families), 62 individuals with a single child with autism 
Table 1 Participant characteristics

\begin{tabular}{|c|c|c|c|c|c|c|c|}
\hline & \multicolumn{3}{|c|}{ Iowa and national samples } & \multicolumn{4}{|c|}{ North Carolina sample } \\
\hline & \multicolumn{3}{|l|}{ Parent groups } & \multicolumn{2}{|l|}{ Parent groups } & \multicolumn{2}{|l|}{ Proband groups } \\
\hline & MIAF $N=215$ & SIAF $N=62$ & DWNS $N=53$ & Autism $N=83$ & Control $N=32$ & Autism $N=36$ & Control $N=38$ \\
\hline Mean age (SD) & $40.2(5.8)$ & $39.9(5.9)$ & $39.6(8.3)$ & $46.6(6.8)$ & $46.2(6.5)$ & $21.5(5.5)$ & $23.3(5.9)$ \\
\hline Male/Female & $106 / 109$ & $40 / 40$ & $30 / 30$ & $37 / 44$ & $13 / 19$ & $29 / 7$ & $30 / 8$ \\
\hline Mean IQ (SD) & $105.2(18.4)$ & $102.8(15.2)$ & $113.1(15.6)$ & $117.5(11.2)$ & $122.1(10)$ & $101.2(18.1)$ & $107.9(15.7)$ \\
\hline
\end{tabular}

MIAF multiple incidence autism family, SIAF single incidence autism family, DWNS Down syndrome family, SD Standard Deviation

(simplex families) and 53 parents of a child with Down syndrome who participated in a family-genetic study of autism and the broad autism phenotype conducted at the University of Iowa (Piven et al. 1997; Losh et al. 2008). The second sample was comprised of a national sample of families, and included 167 parents from multiplex families who participated in the Collaborative Linkage Study of Autism (CLSA 1999). Ascertainment strategies for both groups were comparable and are described in detail elsewhere (CLSA 1999; Piven et al. 1997). Because these two samples included autistic individuals of varying developmental stages and intellectual abilities (IQ cutoff was 235 ), and given that neuropsychological testing of probands was not the subject of these studies, RAN ability was only assessed among parents.

The third sample was collected as part of a family study of the neuropsychological basis of autism and the broad autism phenotype conducted at the University of North Carolina, Chapel Hill. This sample comprised parents $(n=83)$ and high-functioning adults with autism $(n=36)$, as well as their respective control groups (i.e., 32 parent controls and 38 proband controls). Diagnoses of autism were confirmed using ADOS and ADI, as was the case in the first two samples. Controls were screened for family history of autism and related disorders (e.g., fragile X syndrome), and history of brain injury. Groups were matched on age, IQ, and gender (see Table 1). Although family pedigrees were collected, confirming multiple-incidence family status through ADI and ADOS administration with siblings was beyond the scope of this study, and so this information was not available for analysis in the North Carolina sample.

In this sample, RAN was assessed in both the parent and autism groups, and controls. All individuals with autism possessed fluent language abilities and IQs $\geq 80$. Ascertainment strategies differed from the first two samples only in that this study focused on high functioning adolescents and adults with autism, whereas the first two samples included a broader age and ability range of probands. As noted in Analyses, differences across samples were considered in analyses in order to account for such variation in ascertainment and subject characteristics. Further details on this sample are detailed in Losh et al. (2009). Group characteristics for all samples are described in Table 1.

\section{Procedures}

\section{Assessment of rapid automatized naming (RAN)}

RAN was assessed by presenting the color and object naming portions of the Rapid Automatized Naming Task (Denckla and Rudel 1974), which involves naming either a sequence of primary colors (presented as squares) or objects (e.g., chair, pencil) randomly repeated in a series of rows, as rapidly as possible. Scores consist of the number of seconds taken to complete each of the two sets of stimuli (recorded by stopwatch), with lower scores representing better performance. Administration procedures were identical across all three samples.

\section{Assessment of features of the BAP}

The Modified Personality Assessment Schedule-Revised is a semi-structured interview for rating personality characteristics that was adapted from the Personality Assessment Schedule (PAS) (Tyrer 1988) to assess parents of individuals with autism in the Baltimore Family Study (Piven et al. 1994), and has been used in subsequent research to define behavioral and personality styles of the BAP (Piven et al. 1997; Murphy et al. 2000; Losh et al. 2008). Participants are led through a series of questions about themselves and an informant (usually the spouse) is asked similar questions in separate interviews. Ratings are based on behavioral examples given by the subject and/or informants in response to a number of probes. Characteristics are rated either as present $(2)$ or absent $(0,1)$. If the rater believes a trait is present but no behavioral example is elicited from either the subject or informant, it is scored as 1 and considered as absent for the purposes of this study. Personality features assessed and examined in this study included rigid or perfectionistic personality, and socially aloof or untactful personality. These traits are thought to 
parallel the ritualistic/repetitive and social symptom domains of autism, respectively, and have been shown to reliably distinguish autism relatives from controls. Additionally, individuals with the social features of the BAP have been shown to display mild deficits on neuropsychological measures of social cognition (Losh and Piven 2007; Losh et al. 2009).

Based on ratings by at least two independent raters, using concrete examples from subject and informant interviews, eighty-six individuals rated positive for the social features of the BAP, and 74 were rated as positive for the rigid/perfectionistic feature. Kappa coefficients across samples ranged from 0.67 to 1.0 . All raters were naïve to participants' performance on the RAN, although given the varying designs and aims of the studies for which these three samples were tested, not all raters were naïve to autism parent status.

History of language delay was assessed among parents using the Autism Family History Interview. This interview probes early developmental language milestones including age at first word and first phrases, and early reading and spelling difficulties. Given relatively low rates of reports of delay, individuals were considered positive for early language delay if they reported delays in either first words and phrases, or the presence of reading or spelling difficulties in childhood. This resulted in 70 individuals rating positive for history of language-related delays.

\section{Analysis plan}

Performance was indicated by time (number of seconds) to completion, which was compared between the autism parents and controls as well as between individuals with autism and their controls. As noted in the methods, administration procedures were comparable across groups, and parent groups were therefore combined across samples for group comparisons, resulting in 301 parents of individuals with autism and 87 parent controls. Fortyeight parents in the Iowa sample had been the subject of a prior report on RAN (Piven et al. 1997), and were therefore not included in analyses to replicate these earlier findings; however, analyses of associations with BAP features had not previously been performed and so all 349 autism parents were included in those analyses.

Times for color and object naming were combined and analyzed using linear mixed model regression, adjusting for random family effects and potential dataset effects (parent dataset only). In addition, the effect of sex was assessed as an independent predictor and possible confounder. The presence of parent-child associations was investigated using Pearson's correlation coefficient. Of note, complete parental data were not available for all families - 21 individuals with autism were missing data from fathers, and 15 from mothers. Linear mixed models were also used in analyses of associations between RAN and retrospective reports of language delay among parents, adjusting for random family effects and where applicable, fixed dataset effects. All analyses were conduction using SAS version 9.1 (Cary, NC).

\section{Results}

Parents Parents of individuals with autism required significantly more time to complete the RAN (Mean difference $=$ $4.04 \mathrm{~s} ; 95 \%$ CI: $0.82,7.21 ; p=0.0143$ ), adjusted for fixed dataset effects and random family effects. Sex was not an independent predictor, nor did it confound the results. We detected no significant differences between multiplex and simplex families.

Individuals with autism Examining RAN performance among high-functioning individuals with autism also revealed significant differences, with individuals with autism taking significantly more time (Mean difference $=$ $12.66 \mathrm{~s} ; 95 \%$ CI: $3.40,21.91 ; p=0.010)$ than controls. Distributions of the raw data for both parents and probands are presented in Fig. 1.

Parent-child correlations Significant correlations were detected among fathers and their autistic children $(r=0.61$; $p=0.016 ; n=15)$. No significant relationship was observed between mothers and their autistic children $(r=-0.07$; $p=0.775 ; n=21)$. Scatter plots of parent-child performance are presented in Fig. 2a and b.

Associations with features of the broad autism phenotype in parents These exploratory analyses examined associations between RAN and the social and rigid/perfectionistic features of the BAP, features which have been examined previously in relation to neuropsychological functioning among parents of individuals with autism (Losh and Piven 2007; Losh et al. 2009). We additionally examined associations with retrospective reports of language-related delays, which were shown to relate to reading-related tasks in a prior study (Folstein et al. 1999). Both the social personality BAP feature (Mean difference $=4.40 \mathrm{~s} ; 95 \% \mathrm{CI}$ : $1.39,7.41 ; p=0.005$ ) and reports of language delay (Mean difference $=5.23 \mathrm{~s} ; 95 \%$ CI: $1.89,8.57 ; p=0.002$ ) were independently associated with greater time required to complete the RAN. However, rigid/perfectionistic features were not associated with RAN (Mean difference $=1.88 \mathrm{~s}$; 95\% CI: $-1.33,5.09 ; p=0.249$ ).

Associations with autism symptomatology Analyses also explored associations between RAN and autism symptomatology as measured by the Autism Diagnostic Interview, 
Fig. 1 Time to complete the RAN

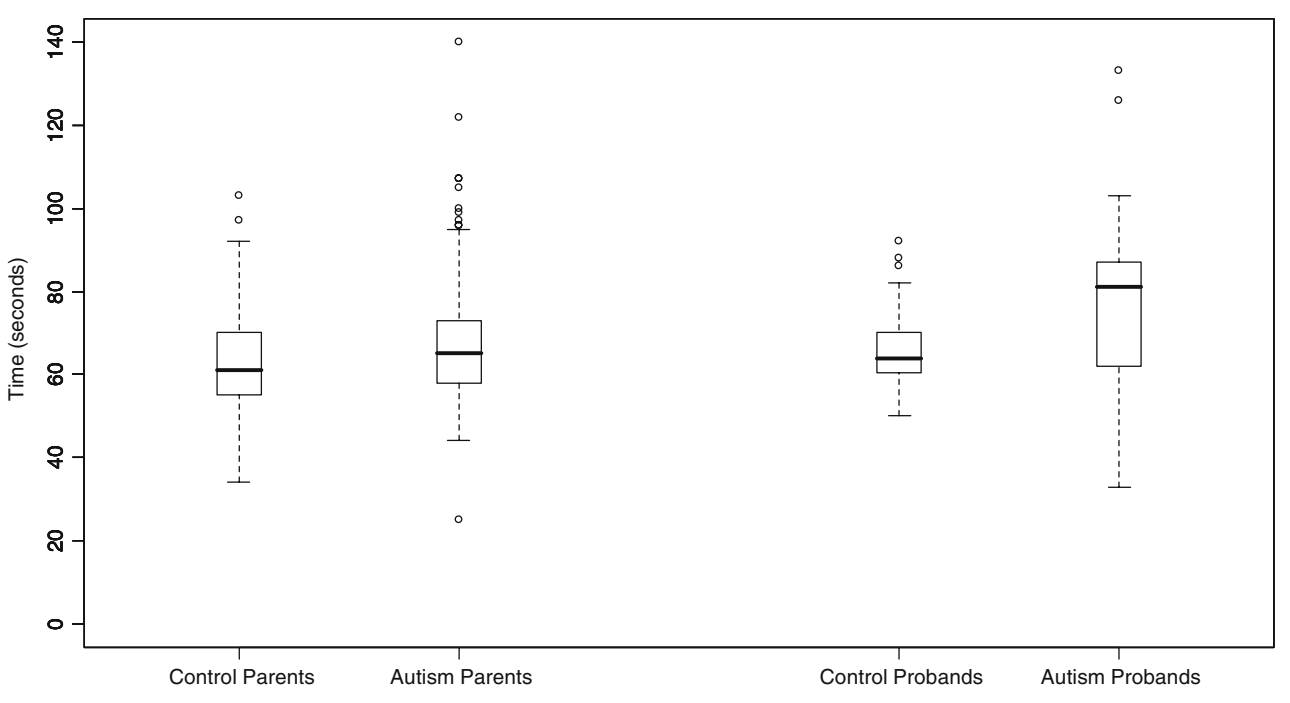

Revised (Lord et al. 1994). No significant associations were detected with any symptom domain assessed by the ADI, or with the total ADI algorithm score; all p-values were at least 0.49 .

\section{Discussion}

The results of this study indicate that high functioning individuals with autism and parents of individuals with autism exhibit deficits in RAN ability. Father-child correlations were also detected, where slower times among fathers were related to similar patterns among children with autism. Finally, results indicated that BAP features among parents were associated with RAN performance, with parents who displayed the social and language characteristics of the BAP demonstrating slower times.

These findings raise several issues relevant to genetic and neurobiological studies of autism. First, parallel patterns of performance detected in autism and in parents of individuals with autism suggest that RAN ability could serve as a marker of latent liability to autism, measurable in both affected and unaffected individuals. Findings that RAN ability was associated with features of the BAP (which are believed to reflect genetic liability) further support this conclusion, as do findings of father-child correlations in performance. The quantitative nature of RAN measurement, along with its demonstrated heritability (Byrne et al. 2002; Petrill et al. 2006) also make this task a potential candidate for inclusion in molecular genetic studies, where the use of quantitative measures (as opposed to categorical variables such as clinical diagnoses) may increase power to detect association and linkage. Indeed, the RAN has been applied in this way in genetic studies of dyslexia with some success (e.g., Wigg et al. 2004).
Findings from this study could suggest that RAN may be applied fruitfully to genetic studies of autism as well.

Second, findings may hold potential for contributing to an emerging literature on indices of neural functioning or processing mechanisms revealing subtle differences in parents of individuals with autism, who do not themselves show evidence of significant psychopathology (e.g., Adolphs et al. 2008). Because the brain regions tapped by RAN have been relatively well-described through lesion and imaging studies, findings may implicate particular neuroanatomical structures mediated by autism susceptibility genes. In particular, RAN ability has been linked to a "reading network" comprised of the inferior frontal cortex, temporo-parietal areas, and the corpus callosum (Dougherty et al. 2007; Geschwind and Fusillo 1966; Misra et al. 2004; Phinney et al. 2006; Pugh et al. 2000; Savage et al. 2007). RAN performance has also been associated with cortical connectivity (Deutsch et al. 2005; Mabbott et al. 2006), which appears to be compromised in autism (Just et al. 2004, 2007; Kana et al. 2009). In a diffusion tensor imaging (DTI) study of autism, Alexander et al. (2007) also demonstrated that reduced fractional anisotropy of the corpus collosum (reflective of lower fiber density, axonal diameter, and myelination) was associated with slower processing speeds on the processing speed index of the Wechsler. Future studies of autism and RAN using DTI and functional neural imaging [feasible with covert RAN methods (Misra et al. 2004)] may prove insightful in pinpointing brain regions and neuroarchitectural features implicated in the performance of individuals with autism and parents. Such studies could also help to illuminate the phenomenology of rapid naming skill, and processing speed in general.

Finally, findings that RAN performance was associated with features of the BAP provide additional converging 
Fig. 2 a Mother-child correlations. b Father-child correlations
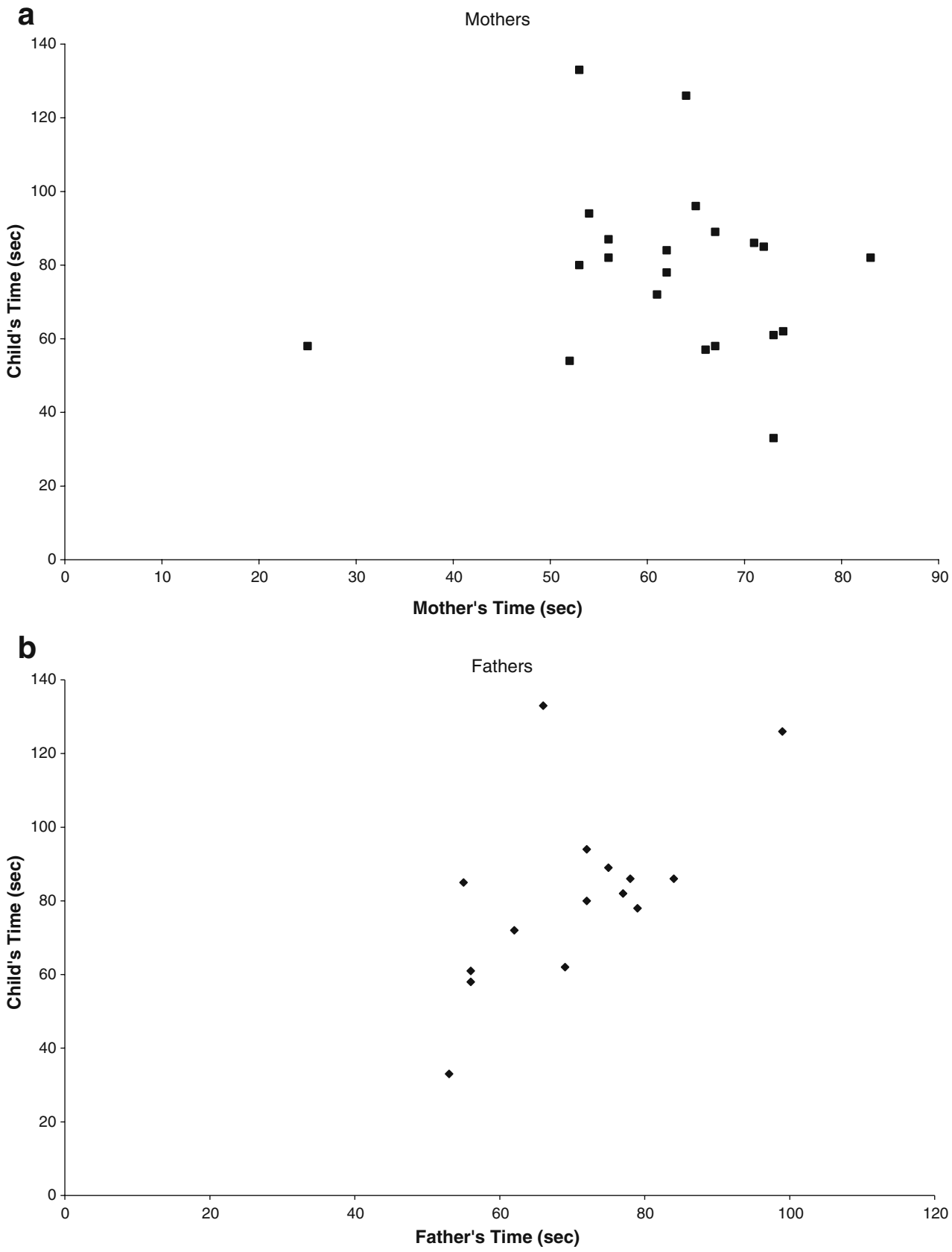

evidence to support the validity of the BAP. As an index of a number of cognitive and verbal processes, RAN could inform the underpinnings of these subtle clinical phenomena. In particular, slower times completing RAN were associated with the social features of the BAP (aloof or untactful) as well as with history of developmental language delays. RAN ability involves a confluence of language and executive control processes that could explain such associations. For instance, RAN performance has been shown to relate to attention, articulation, planning, inhibition, and lexical memory and retrieval abilities (Bowers and Wolf 1993; Clarke et al. 2005; Lervag and Hulme 2009; Wolf et al. 2000), each of which may conceivably relate to a history of developmental language delays. Ties between such abilities and the social features of the BAP, however, are not as straightforward and could indicate that the social and language features of the BAP constitute variable manifestations of one or more common underlying mechanisms. This possibility is consistent with findings from a recent factor analysis indicating that some language and social features of the BAP loaded on the same factor (Losh et al. 2008). The lack of association with the rigid/perfectionistic feature of the BAP is consistent with our prior study of the neuropsychological basis of autism and the BAP, in which only those parents displaying the social features of the BAP showed differences from controls (Losh et al. 2009). 
Because associations between RAN and the BAP were exploratory, the current findings need to be replicated in future studies. And, whereas results were robust statistically, it is important to note that actual mean group differences among parent groups were relatively small (with approximately $4 \mathrm{~s}$ differentiating autism parents from controls). Differences from controls and associations with features of the BAP are therefore unlikely to reflect clinically significant impairment, but rather, may be more accurately characterized as constituting a subtle cognitive-linguistic marker of genetic liability to autism, akin to smooth pursuit eye movement differences documented among relatives of individuals with schizophrenia (Clementz and Sweeney 1990).

The lack of significant associations between RAN and domains of autism symptomatology should also be investigated further. It could be that when all three domains of autism are present to a degree sufficient to warrant a diagnosis of autism, more subtle domain-specific associations (as evident in the BAP) become obscured. The study may also have lacked adequate power to detect associations in the autism group.

A final question for future studies concerns the finding that parent-child correlations in RAN ability were evident in father-child dyads only. It is worth noting that significant associations were detected in a sample of only 15 father-child pairs, suggesting that this effect is relatively robust for fathers. Perhaps such effects among mothers are more subtle, rendering the current sample of 21 mother-child pairs insufficient. Larger samples of intact families would address this important question and also help to inform models of intrafamilial transmission that will be important to consider in genetic studies of autism. Together with further study of RAN in relation to BAP features, such work may contribute to knowledge of the neurobiological, and ultimately genetic significance of RAN in autism and the BAP.

Acknowledgments This project was funded by STAART Center Grant \#1 U54 MH66418, R01 MH055284, K20 MH001028, K02 MH001568, and R29 MH051217 to JP. ML acknowledges support from Autism Speaks and the National Science Foundation (\# 0820394). ML and DE acknowledge support from CTSA UL1RR025747.

\section{References}

Adolphs R, Spezio M, Parlier M, Piven J. Distinct face-processing strategies in parents of autistic children. Curr Biol. 2008;18 (14):1090-3.

Alexander AL, Lee JE, et al. Diffusion tensor imaging of the corpus callosum in Autism. Neuroimage. 2007;34(1):61-73.

APA: Diagnostic and statistical manual of mental disorders (DSM-IV), 4 ed. Washington, DC; 1994.

Bailey A, Le Couteur A, Gottesman I, Bolton P, Simonoff E, Yuzda E, et al. Autism as a strongly genetic disorder: evidence from a British twin study. Psychol Med. 1995;25:63-77.
Barrett S, Beck JC, et al. An autosomal genomic screen for autism. Collaborative linkage study of autism. Am J Med Genet. 1999;88 (6):609-15.

Bishop DV, Maybery M, Wong D, Maley A, Hill W, Hallmayer J. Are phonological processing deficits part of the broad autism phenotype? Am J Med Genet B Neuropsychiatr Genet. 2004;128B(1):54-60.

Bolton P, Macdonald H, Pickles A, Rios P, Goode SCM, Bailey A, et al. A case-control family history study of autism. J Child Psychol Psychiatry. 1994;35:877-900.

Bowers PG, Wolf M. Theoretical links among naming speed, precise timing mechanisms and orthographic skill in dyslexia. Reading and Writing, an Interdisciplinary Journal. 1993;5(1):69-85.

Byrne A, MacDonald J, et al. Reading, language and memory skills: a comparative longitudinal study of children with Down syndrome and their mainstream peers. Br J Educ Psychol. 2002;72(Pt 4):513-29.

Clarke P, Hulme C, et al. Individual differences in RAN and reading: a response timing analysis. J Res Read. 2005;28(2):73-86.

Clementz BA, Sweeney JA. Is eye movement dysfunction a biological marker for schizophrenia? A methodological review. Psychol Bull. 1990;108:77-92.

Compton DL, Davis CJ, DeFries JD, Gayçn J, Olson RK. Genetic and environmental influences on reading and RAN: An overview of results from the Colorado twin studies. In: Wolf $\mathrm{M}$, editor. Dyslexia, fluency, and the brain Timonium. MD: York; 2001.

de Kovel CG, Franke B, Hol FA, Lebrec JJ, Maassen B, Brunner H, et al. Confirmation of dyslexia susceptibility loci on chromosomes $1 \mathrm{p}$ and $2 p$, but not $6 p$ in a Dutch sib-pair collection. Am J Med Genet B Neuropsychiatr Genet. 2008;147(3):294-300.

Denckla MB. Color-naming defects in dyslexic boys. Cortex. 1972;8:164-76.

Denckla MB, Rudel R. Rapid "automatized" naming of pictured objects, colors, letters and numbers by normal children. Cortex. 1974; 10:186-202.

Denckla MB, Rudel RG. Rapid "automatized" naming (R.A.N.): Dyslexia differentiated from other learning disabilities. Neuropsychologia. 1976;14:471-9.

Denckla MB, Cutting LE. History and significance of rapid automatized naming. Ann Dyslexia. 1999;49:29-42.

Deutsch GK, Dougherty RF, et al. Children's reading performance is correlated with white matter structure measured by diffusion tensor imaging. Cortex. 2005;41(3):354-63.

Dougherty R, Ben-Shachar M, Deutsch G, Hernandez A, Fox G. Temporal-callosal pathway diffusivity predicts phonological skills in children. Proc Natl Acad Sci. 2007;104(20):8556-61.

Folstein S, Rutter M. Infantile autism: a genetic study of 21 twin pairs. J Child Psychol Psychiatry. 1977;18(4):297-321.

Folstein SE, Santangelo SL, Gilman SE, et al. Predictors of cognitive test patterns in autism families. J Child Psychol Psychiatry. 1999;40(7):1117-28.

Geschwind N, Fusillo M. Color-naming defects in association with alexia. Arch Neurol. 1966;15:137-46.

Grigorenko EL, Wood FB, Meyer MS, Hart LA, Speed WC, Shuster A, et al. Susceptibility loci for distinct components of developmental dyslexia on chromosomes 6 and 15. Am J Hum Genet. 1997;60(1):27-39.

Grigorenko EL, Wood FB, Meyer MS, Pauls JE, Hart LA, Pauls DL. Linkage studies suggest a possible locus for developmental dyslexia on chromosome 1p. Am J Med Genet. 2001;105(1):120-9.

Holland J, McIntosh D, Huffman L. The role of phonological awareness, rapid automization, and orthographic processing in reading. J Psychoeduc Assess. 2004;22:233-60.

Hughes C, Plumet MH, Leboyer M. Towards a cognitive phenotype for autism: increased prevalence of executive dysfunction and superior spatial span amongst siblings of children with autism. J Child Psychol Psychiatry. 1999;40(5):705-18. 
Just MA, Cherkassky VL, Keller TA, Minshew NJ. Cortical activation and synchronization during sentence comprehension in highfunctioning autism: evidence of underconnectivity. Brain. 2004; 127:1811-21.

Just MA, Cherkassky VL, Keller TA, Kana RK, Minshew NJ. Functional and anatomical cortical underconnectivity in autism: evidence from an FMRI study of an executive function task and corpus callosum morphometry. Cereb Cortex. 2007;17(4):951-61.

Kana R, Keller T, Cherkassky V, Minshew N, Just M. Atypical frontalposterior synchronization of Theory of Mind regions in autism during mental state attribution. Soc Neurosci. 2009;4(2):135-52.

Lervåg $\mathrm{A}$, Hulme $\mathrm{C}$. Rapid automatized naming (RAN) taps a mechanism that places constraints on the development of early reading fluency. Psychol Sci. 2009;20(8):1040-8.

Lindgren KA, Folstein SE, et al. Language and reading abilities of children with autism spectrum disorders and specific language impairment and their first-degree relatives. Autism Res. 2009;2:22-38.

Lord C, Rutter M, Le Couteur A. Autism diagnostic interview-revised: a revised version of a diagnostic interview for caregivers of individuals with possible pervasive developmental syndromes. Journal of Autism and Developmental Syndromes. 1994;24:659-85.

Lord C, Risi S, Lambrecht L, Cook EH, Leventhal BL, DiLavore PC, et al. The autism diagnostic observation schedule-generic: a standard measure of social and communication deficits associated with the spectrum of autism. J Autism Dev Disord. 2000;30:205-23.

Losh M, Piven J. Social-cognition and the broad autism phenotype: identifying genetically meaningful phenotypes. J Child Psychol Psychiatry. 2007;48:105-12.

Losh M, Childress D, Lam K, Piven J. Defining key features of the broad autism phenotype: a comparison across parents of multiple-and single-incidence autism families. American Journal of Medical Genetics, Psychiatric Genetics. 2008;147B:424-33.

Losh M, Adolphs R, Poe M, Penn D, Couture S, Baranek G, et al. The Neuropsychological profile of autism and the broad autism phenotype. Arch Gen Psychiatry. 2009;66:518-26.

Losh, M., R. Adolphs, et al. The broad autism phenotype. Autism Spectrum Disorders. G. Dawson, D. Amaral and D. Geschwind, Oxford University Press; 2010.

Mabbott DJ, Noseworthy M, Bouffet E, Laughlin S, Rockel C. White matter growth as a mechanism of cognitive development in children. Neuroimage. 2006;33(3):936-46.
Misra M, Katzir T, et al. Neural systems for rapid automatized naming in skilled readers: unraveling the RAN-reading relationship. Sci Stud Read. 2004;8(3):241-56.

Murphy M, Bolton P, et al. Personality traits of the relatives of autistic probands. Psychol Med. 2000;30(6):1411-24.

Nopola-Hemmi J, Myllyluoma B, Voutilainen A, Leinonen S, Kere J, Ahonen T. Familial dyslexia: neurocognitive and genetic correlation in a large Finnish family. Dev Med Child Neurol. 2002;44(9):580-6.

Petrill SA, Thompson LA, Deater-Deckard K, Dethorne LS, Schatschneider $\mathrm{C}$. Genetic and environmental effects of serial naming and phonological awareness on early reading outcomes. J Educ Psychol. 2006;98(1):112-21.

Phinney E, Pennington BF, Olson R, Filley CM, Filipek PA. Brain structure correlates of component reading processes: implications for reading disability. Cortex. 2006;43(6):777-91.

Pilowsky T, Yirmiya N, et al. Language abities of siblings of children with autism. J Child Psychol Psychiatry. 2003;44:914-25.

Piven J, Palmer P. Cognitive deficits in parents from multiple incidence autism families. J Child Psychol Psychiatry. 1997;38:1011-21.

Piven J, Wzorek M, et al. Personality characteristics of the parents of individuals with autism. Psychol Med. 1994;24(3):783-95.

Piven J, Palmer P, Landa R, Santangelo S, Jacobi D, Childress D. Personality and language characteristics in parents from multipleincidence autism families. Am J Med Genet (Neuropsychiatric Genetics). 1997;74:398-411.

Pugh KR, Mencl WE, Jenner AR, Katz L, Frost SJ, Lee JR, et al. Functional neuroimaging studies of reading and reading disability (developmental dyslexia). Ment Retard Dev Disabil Res Rev. 2000;6(3):207-13.

Savage R, Pillay V, Melidona S. Deconstructing rapid automatized naming: component processes and the prediction of reading difficulties. Learn Individ Differ. 2007;17(2):129-46.

Smalley SL, Asarnow RF. Cognitive subclinical markers of autism. J Autism Dev Disord. 1990;20(2):271-8.

Tyrer P. Personality assessment schedule. In: PDDMa, editor. Course. London: Butterworth; 1988.

Wigg KG, Couto JM, Feng Y, Anderson B, Cate-Carter TD, Macciardi F, et al. Support for EKN1 as the susceptibility locus for dyslexia on 15q21. Mol Psychiatry. 2004;9(12):1111-21.

Wolf M, Bowers PG, Biddle K. Naming-speed processes, timing, and reading: a conceptual review. J Learn Disabil. 2000;33(4):387-407. 